\title{
PEST MANAGEMENT USING MACHINE LEARNING ALGORITHMS: A REVIEW
}

\section{R. P. L. DURGABAI, P. BHARGAVI \& S. JYOTHI}

Department of Computer Science, Sri Padmavati Mahila Visva vidyalayam, Tirupati, Andhra Pradesh, India

Agriculture is a unique business crop production, which is dependent on many climate and economy factors, the major occupation of Indians is farming where in about $70 \%$ of the population depends on agriculture. Farmers have wide range of diversity to select suitable Fruit and Vegetable crops. However, the cultivation of these crops for optimum yield and quality produce is highly technical. The crop production has reduced due to various factors like pest attack, diseases and climatic conditions. Crop protection is the science and practice of managing pests, plant diseases and other pest organisms that damage agricultural crops. Machine learning is a looming field of computer science which can be applied to the farming sector quite effectively. It can facilitate the up-gradation of conventional farming techniques in the most cost-friendly approach. This paper reviews on how different machine learning algorithms are useful in pest management of various crops.
\end{abstract}

KEYWORDS: Pest Management, Machine Learning Algorithms \& Agriculture

Received: Nov 28, 2017; Accepted: Dec 18, 2017; Published: Jan 06, 2018; Paper Id.: IJCSEITRFEB20182

\section{INTRODUCTION}

Agriculture is the main source of the Indian Economy and about 70 per cent of India's rural population are dependent on agriculture for their livelihood. Agriculture is one of the main contributors to the Gross Domestic Product in India. Sustainable agriculture, in terms of food security, rural employment, and environmentally sustainable technologies such as soil conservation, sustainable natural resource management and biodiversity protection, are essential for holistic rural development. India's agriculture is composed of many crops, with the foremost food staples being rice and wheat. Indian farmers also grow pulses, potatoes, sugarcane, oilseeds, and such non-food items as cotton, tea, coffee, rubber, and jute [31]. However, the major loss in agriculture profit is observed mainly due to improper Pest management and lack of awareness in Information Technology.

Managing pests (weeds, insects and plant diseases) in agriculture involves the safe and environmentally sound use of pesticides to control crop pests when and where needed, as well as Integrated Pest Management (IPM) strategies that avoid total reliance on chemical pesticides. IPM is the most comprehensive way to manage pests. It is a set of strategies based on monitoring, economic thresholds and preventive tactics to determine if and when pest treatment is needed. IPM strategies are presently better developed for insect control than weed control. IPM is especially advanced for fruit and vegetable production [32].

To detect a plant pest in very initial stage, use of Machine Learning technique is beneficial. Machine learning is an emerging technology that can aid in the discovery of rules and patterns in sets of data. It has frequently been observed that the volume of recorded data is growing at an astonishing rate that far outstrips our ability to make sense of it. The "database mining" is now being used to describe efforts to analyze data sets automatically for significant structural regularities. Potential applications of these techniques are applied in the 
domains such as agriculture. In recent years more emphasis is given to research studies on the relevance of machine learning techniques in the domain of agricultural pest management. This paper presents on machine learning algorithms that are applied in the domain of pest management in agriculture, such as artificial neural networks, Support Vector Machines, Decision Tree, Regression Analysis, Bayesian belief network [6].

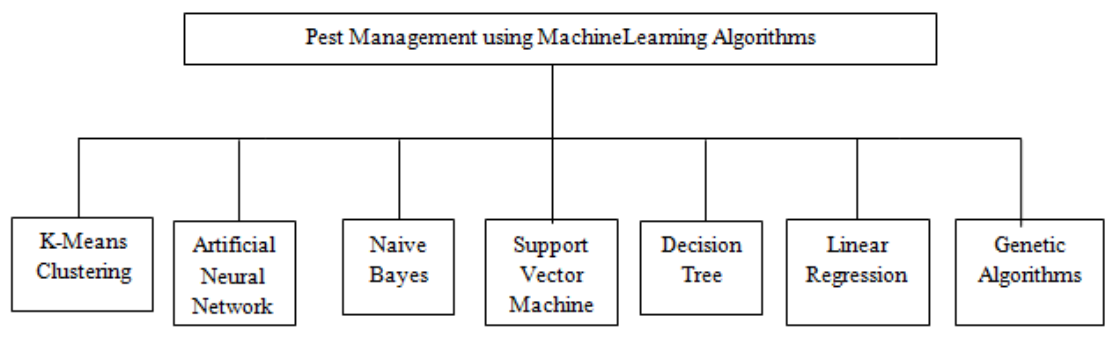

\section{PEST MANAGEMENT}

Recent research efforts have focused on predicting diseases and crop pest using computer science techniques such as Machine learning algorithms. Plant pathogens and crop-feeding insects are integral part of agro ecosystems. A first group is related to pathogen population dynamics, and concerns the dynamics of Pests and Disease Models (PDM), through which populations may spatially expand and temporally increase. The second group addresses crop losses, and focuses on the consequences of the host-pathogen interactions on crop physiological processes and yield. Pest management will be of crucial importance, given that crop yield losses caused by weeds (about 32\%) are higher than those caused by other pests $(18 \%)$ or pathogens $(15 \%)$. The studies of plant trait/disease refer to the studies of visually observable patterns of a particular plant.

Nowadays crops face many traits/diseases. Damage of the insect is one of the major trait/disease. Insecticides are not always proved efficient because insecticides may be toxic to some kind of birds. It also damages natural animal food chains. A common practice for plant scientists is to estimate the damage of plant (leaf, stem) because of disease by an eye on a scale based on percentage of the affected area. In most of the cases, pests or diseases are seen on the leaves or stems of the plant. Therefore, identification of plants, leaves, stems and finding out the pest or diseases, the percentage of the pest or disease incidence, symptoms of the pest or disease attack, plays a key role in the successful cultivation of crops. IPM is the most comprehensive way to manage pests. It is a set of strategies based on monitoring, economic thresholds and preventive tactics to determine if and when pest treatment is needed. IPM strategies are presently better developed for insect control than weed control. IPM is especially advanced for fruit and vegetable production [32].

IPM is not a single pest control method, but, rather, a series of pest management evaluations, decisions and controls. In practicing IPM, growers who are aware of the potential for pest infestation follow a four-tiered approach. The four steps include:

\section{- $\quad$ SetActionThresholds}

Before taking any pest control action, IPM first sets an action threshold, a point at which pest populations, or environmental conditions indicate that pest control action must be taken. Sighting a single pest has not always meant that control is needed. The level at which pests will either become an economic threat is critical to guide future pest control decisions. 


\section{- Monitor and Identify Pests}

All insects, weeds, and other living organisms need not require control. Many organisms are innocuous, and some are even beneficial. IPM programs work to monitor for pests and identify them accurately, so that appropriate control decisions can be made in conjunction with action thresholds. This monitoring and identification removes the possibility that pesticides will be used when they are not really needed or that the wrong kind of pesticide is used.

\section{- Prevention}

As a first line of pest control, IPM programs work to manage the crop, lawn, or indoor space to prevent pests from becoming a threat. In an agricultural crop, this may mean using cultural methods, such as rotating between different crops, selecting pest-resistant varieties, and planting pest-free root stock. These control methods can be very effective and costefficient and present little to no risk to people or the environment.

\section{- Control}

Once monitoring, identification, and action thresholds indicate that pest control is required, and preventive methods are no longer effective or available, then IPM programs evaluate the proper control method both for effectiveness and risk. Effective, less risky pest controls are chosen first, including highly targeted chemicals, such as pheromones to disrupt pest mating, or mechanical control, such as trapping or weeding. Further monitoring, identifications and action thresholds indicate that less risky controls are not working, then additional pest control methods would be employed, such as targeted spraying of pesticides. Broadcast spraying of non-specific pesticides is a last resort.

\section{MACHINE LEARNING ALGORITHMS}

Machine learning is the branch of computer science which is used to construct algorithms which exhibit selflearning property, i.e. learning which is done by the machine itself hence the term 'Machine Learning'. It is considered to be one of the major areas under Artificial Intelligence. For a machine to become intelligent like a human mind, it has to first think and learn like a human. Human mind learns from past data and experiences that it is exposed to and based on that it takes decisions in future. Any conventional computer algorithm works as it is programmed by its developer. In other words, it will only follow the instructions given by its controller. For a machine to exhibit intelligence, it has to interpret and analyze the input and result data apart from simply following the instructions on that data. This is what the machine learning algorithms do.

These algorithms can build the system model from the input and output data. The system can then be used to predict future values. These methods are far better than the conventional statistical methods as they do not rely on the userspecified parameters rather self improvises using the available data. The applications of machine learning are multidisciplinary where traditional rule-based algorithms are not possible to be constructed or fail to deliver correct results. One such field is agriculture. There are different types of algorithms in machine learning as follows:

\section{- $\quad$ K-Means Clustering}

K-means is one of the simplest machine learning algorithms that solve the well known clustering problem. The procedure follows a simple and easy way to classify a given data set through a certain number of clusters (assume $\mathrm{k}$ clusters) fixed a priori. The main idea is to define $\mathrm{k}$ centroids, one for each cluster. These centroids should be placed in a cunning way because of different location causes different result. So, the better choice is to place them as much as possible 
far away from each other [33].

\section{- Artificial Neural Networks}

Artificial Neural network (ANN) is an interconnected set of input and output units where a weight is associated with each connection. It is based on the structure and functions of biological neural networks. It works like the way human brain processes information. It includes a large number of connected processing units that work together to process information. They also generate meaningful results from it [34].

\section{- Naive Bayes}

Naive Bayes is a simple but surprisingly powerful algorithm for predictive modelling. The representation used by naive Bayes that is actually stored when a model is written to a file. One of the easiest ways of selecting the most probable hypothesis given the data that we have that we can use as our prior knowledge about the problem. Bayes' Theorem provides a way that we can calculate the probability of a hypothesis given our prior knowledge [35].

\section{- $\quad$ Support Vector Machine}

In machine learning, Support Vector Machines (SVMs, also support vector networks are supervised learning models with associated learning algorithms that analyze data used for classification and regression analysis [36].

\section{- Decision Tree}

A tree has many analogies in real life, and turns out that it has influenced a wide area of machine learning, covering both classification and regression. In decision analysis, a decision tree can be used to visually and explicitly represent decisions and decision making. As the name goes, it uses a treelike model of decisions [37].

\section{- Linear Regression}

Linear regression was developed in the field of statistics and is studied as a model for understanding the relationship between input and output numerical variables, but has been borrowed by machine learning. It is both a statistical algorithm and a machine learning algorithm [38].

\section{- Genetic Algorithms}

Genetic algorithms are stochastic search algorithms which act on a population of possible solutions. They are loosely based on the mechanics of population genetics and selection. The potential solutions are encoded as 'genes', strings of characters from some alphabet. New solutions can be produced by 'mutating' members of the current population, and by 'mating' two solutions together to form a new solution. The best solutions are selected to breed and mutate and the worse ones are discarded. They are probabilistic search methods; this means that the states which they explore are not determined solely by the properties of the problems [39].

\section{MACHINE LEARNING ALGORITHMS FOR PEST MANAGEMENT}

Machine learning methods have been used in the recent years for crop disease prediction and these efforts have been proved worthwhile. They revealed higher accuracy compared to the traditional statistical methods like regression analysis. These methods deal well with noisy and multi-faceted data. Early crop disease detection and classification has been done using Support Vector Machines. There are several factors like soil quality, crop rotation cycle, seed quality 
etwhich can lead to poor health and diseases in crops. Machine learning algorithms effectively take into consideration all the possible factors, historic data as well as satellite/sensor data of fields to provide valuable disease classifiers. Disease detection using images of crop leaves has been implemented using a pattern recognition branch of machine learning. It works by obtaining patterns of input data and separating them into classes of diseases. Jayamala K. Patil, et al., [1] detected plant diseases using machine-learning methods that achieved, speed and accuracy. Hence there is a scope for working on the development of innovative, efficient $\&$ fast interpreting algorithms which will help plant scientist in detecting disease.

To Identify and classify the diseases in turmeric leaf Nandhini M et al., [2] used Support Vector Machine, Decision Tree, Naive Bayes to identify and classify the diseases in turmeric leaf. Multidisciplinary research approach of integrating computational with agriculture will help in forecasting pest managing agricultural crops efficiency [3]. P. Revathi et al., The treatment for the diseases effected to the chilly plants are defined by using rule based systems and Artificial Bee Colony, by applying machine learning techniques resulting to best global optimized solutions for recognizing the diseases in chilly plants.

DavidCamilo Corrales et al., [4] defined the detection of diseases and pests in different crops using supervised learning algorithms. The Research and algorithms were compared in order to observe the performance. Decision Tree is being regarded as the most widely used and the best to generate easily interpretable classifiers followed by Support Vector Machine and Artificial Neural Networks algorithms for predicting or classifying diseases and pests. Arti Singh et al., [5] Machine learning in Identification, Classification, Qualification and Prediction (ICQP) is being used for plant stress phenol typing. The Machine Learning tools which provide a very powerful framework to assimilate data and the utility of these tools is especially important considering current progress in HTP approaches that easily generate terabytes of data. Subhadra Mishra et al., [6] stated the effort on the relevance of machine learning techniques in the domain of agriculture crop production. The Prediction of crop can be performed by using various Machine learning algorithms such as mathematical and statistical method etc.

The improvement and application of pest and disease models to analyze and predict yield losses, including those due to climate change is still a challenge for the scientific community M. Donatelli et al., [7]. The need to estimate the impact of pests and diseases on agricultural production is an important element in the development and analysis of scenarios impacting farmers' income and food security. Suraksha I et al., [8] implements an innovative idea to identify diseases affected in paddy crops and provides the remedy and solution to protect crops. The images are then fed to application for identification of paddy diseases and the remedies are suggested to the farmer. This implementation providesthe bestchoice for every class of agriculture community, particularly in remote villages.

F K, Van Evert et al., [9] used a new conceptual model for weed control and crop protection to develop which consists of three elements: (i) capture and store data, (ii) analyze data and (iii) generate recommendations. Integrated solutions for weed control and crop protection are needed. Georg Rub et al.,[10] After Data Collection, Analysis, Recommendation predicting wheat yield from cheaply-available in-season data is being done using Neural Networks. Sjaak Wolfert et al., [11] analyzed that the Big Data is changing the scope and organization of farming through a pull-push mechanism such as food security and safety, sustainability. Karandeep Kaur [12] Organized to broaden the gaming horizon by listing and evaluating the different applications of machine learning in Indian agriculture and to help the farmers advance their work up by many notches. Vijay Singh et al., [13] Different diseases classification techniques are being used 
for plant leaf disease detection. An algorithm for image segmentation technique is being used for automatic detection as well as classification of plant leaf diseases later.

Yun Hwan Kim et al., [14]In the prediction of crop pests, Machine learning technology like SVM (Support Vector Machine), Multiple Linear Regression, Neural Network, and Bayesian Network based techniques, are being used for current trends. SaeedAzfaret.al[15] Wireless Sensor Networks is being used for Pest control mechanism based on their effectiveness, cost and other performance parameters. Jiangui Liuet al.,[16] Inverted Gaussian Model is applied to study the relationships between crop variables and the red edge parameters. A. K. Tripathy et al., [17] conducted an experiment in a semi-arid region of India to understand the crop-weather-pest relations using wireless sensors and field-level surveillance data on the groundnut pest Trips. Data mining techniques were used to turn the data into useful information and correlation of crop-weather-pest field. Dr. Navneet Goyal [18]used a classification system which is developed for quality grading of mushrooms. D Ramesh et al.,[19]using Data mining techniques Yield prediction can be solved based on the available data as it is a very important agricultural Problem. Sally Jo Cunningham et al., [20]Machine learning algorithms like Classifiers, Naive Bayes, Decision Table is being used to mine information from agricultural data sets and to perform basic research in data mining. Jay Gholap et al., [21] analyzed that soil dataset using data mining techniques, an automated system for soil classification has been developed based on fertility. SitiKhairunniza - Bejo et al., [22] The use of Artificial Neural Network (ANN) in predicting crop yield using various crop performance factors is being used to identify any interactions between factors that affects yield with the crop Performance.

SidikMulyonoet al., [23] MODIS (Moderate Resolution Imaging Spectro radiometer)along with Remote sensing images with Support Vector Machines are being used to classify paddy growth stages. Manpreet Kauretal., [24] used Data mining techniques to solve the problem of Price Prediction of Crops based on the available data which has become a very important agricultural problem. Hotal Pateletal., [25] Predictive data mining technique is being used to predict future crop, weather forecasting, pesticides, and fertilizers. Prediction involves using some variables or fields in the database to predict unknown or future values of other variables of interest.

ROBERT J. McQUEEN et al., [26] Machine learning strategies have been applied to overcome the problems in agriculture and horticulture. Similarity based techniques have been used in the domain of agriculture. Jharna Majumdar et al., [27]Data mining techniques have been used to analyze the agriculture data, DBSCAN method is being used to cluster the data, based on districts which are having temperature, rainfall and soil type. Elpiniki I et al., [28] Soft computing technique of Fuzzy Cognitive Maps is applied with an efficient unsupervised learning algorithm to describe the cotton yield management in precision farming. Latika Sharma et al., [29] Data Mining technique is being used as a tool for knowledge management to bring out the characteristic computational needs of agriculture data which is essentially seasonal ,and uncertain. SalvatoreRampone et al., [30] usedtwo Soft computing methods such as Artificial Neural Networks and Multilayer Perceptron approach and Genetic Programming are applied to Predict next year's seasonal temperatures. M. G. Hill et al., [40] Using industry spray diary and pest monitoring data gathered at an orchard block level for compliance purposes, 80 attributes (independent variables) were created in three categories from the spray diary data: (1) individual insecticide applications applied during 2-week time windows, (2) groups of insecticide applications within time periods prior to or after fruit set and (3) orchard management attributes. Five machine learning algorithms (Decision Tree, Naïve Bayes, Random Forest, AdaBoost, Support Vector Machine) and one statistical method (Logistic regression) (classifiers) were used to develop models to forecast insecticide application decisions for leaf roller control, by predicting 
whether pest monitoring results were above or below a spray threshold. Oladapo J. Olakulehin et al., [41] A genetic algorithm approach has been proposed to determine the crop yield maximization while sustaining soil fertility. Veerle Desmet et al., [42] how decision trees being used to improve the Ball and Larus heuristics by optimizing the sequence of applying the heuristics and by discovering two new heuristics, namely one based on the post domination relationship between the current basic block and its successor and one based on the dependency distance between the branch and its operand defining instruction. Amie L. Melnychuk et al., [43] To determine the best image timing for crop classification a comparison of two supervised classifications using optical satellite imagery with multiple single-date classifications and a subsequent multi-date, multi-sensor classification has been used. KefayaQaddoum [44] To model a simple but often satisfactory supervised classification method utilized regularization technique is being used to obtain a computationally efficient classifier based on Naive Bayes. The suggested construction, utilized L1-penalty, is capable of clearing redundant predictors, where a modification of the LARS algorithm is devised to solve this problem, making this method applicable to a wide range of data. L. Peacock et al., [45] To predict the geographic distribution of groups of polyphagous plant pests, artificial neural networks has been used, using climate variables as predictors, artificial neural network models, were compared with binary logistic models for predicting insect distribution.

\section{CONCLUSIONS}

This Paper reviews on Crop, Pest attacks and diseases, different computing techniques on pests and Machine learning algorithmswere discussed. The survey is summarized on various aspects of agriculture. And the Hierarchical chart in introduction gives information about various machine learning techniques applied to pest management. The machine learning algorithms can help farmers in predicting the pest attack depends on aspects like climate, soil, temperature and increase their income by reducing damages.

\section{REFERENCES}

1. Jayamala, K., Patil, Raj Kumar, June 2011. "Advances In Image Processing For Detection Of Plant Diseases", Journal of Advanced Bioinformatics Applications and Research ISSN 0976-2604Vol 2, Issue 2, pp 135-141.

2. Nandhini, M., PreamSudha, V., Vijaya, MS., February 2016. "Identification and classification of leaf diseases in Turmeric Plants", Int. Journal of Engineering Research and Applications,www.ijera.com ISSN: 2248-9622, Vol. 6, Issue 2, (Part - 2), pp.48-54.

3. Revathi, P., Revathi, R., Hemalatha, M., 2011. "Comparative study of knowledge in Crop diseases using Machine Learning Techniques", IJCSIT International Journal of Computer Science and Information Technology,vol.2(5),,2180-2182,ISSN:09759646.

4. Corrales, D. C., June, 2015. "Towards Detecting Crop Diseases and Pest by supervised learning", Univ., vol. 19, no. 1, pp. 207-228.

5. Arti Singh, BaskarGanapathysubramanian, Asheesh Kumar Singh, SoumikSarkar,February 2016. Trends in Plant Science," Machine Learning for High-Throughput Stress Phenotyping in Plants ". Published by Elsevier Ltd., Vol. 21, No. 2.

6. Subhadra Mishra, Debahuti Mishra, GourHariSantra, October 2016. "Applications of Machine Learning Techniques in Agricultural Crop Production : A Review Paper", Indian Journal of Science \& Technology, Volume 9, Issue 38.

7. Donatelli, M., Magarey, R.D.,Bregaglio, S., Willocquet, L., Whish, J.P.M., Savary, S., February 2017. "Modelling the impacts of pests and diseases on agricultural systems", Published by Elsevier Ltd. 
8. Suraksha, I., Sushma, B., Sushma, R.G., SushmithaKeshav, Uday Shankar, S, V., May 2016. "Disease Prediction of Paddy Crops Using Data Mining and Image Processing Techniques", International Journal of Advanced Research in Electrical, Electronics and Instrumentation Engineering,Vol. 5, Issue 5.

9. Van Evert, F, K., Fountas, S., Jakovetic, D., Crnojevic, V., Travlos, I., Empenaar, C., March 2017. "Disease Prediction of Big Data for weed control and crop protection", An International Journal of weed Biology,Ecology and Vegetation Management, Weed Research published by John Wiley \& Sons Ltd on behalf of European Weed Research Society. 57, 218-233.

10. Georg Rub, Rudolf Kruse, Martin Schneider, Peter Wagner, 2008."Data Mining with Neural Networks for Wheat Yield Prediction", Industrial Conference on Data Mining, pp 47-56.

11. SjaakWolfert, LanGe, CorVerdouw, Marc-JeroenBogaardt, February 2017. "Big Data in Smart Farming - A review"- journal homepage: www.elsevier.com/locate/agsy

12. KarandeepKaur, 2016. "Machine Learning: Applications in Indian Agriculture", International Journal of Advanced Research in Computer and Communication Engineering Vol. 5, Issue 4, April 2016, ISSN (Online) 2278-1021 ISSN (Pint) 23195940.

13. VijaiSingh,A.K. Misra, November 2016, "Detection of plant leaf diseases using image segmentation and soft computing techniques",INFORMATION PROCESSING IN AGRICULTURE 4 (2017) 41-49.

14. Yun Hwan Kim, SeongJoonYoo, YeongHyeonGu, Jin Hee Lim, Dongil Han, Sung WookBaik,2013."Crop Pests Prediction Method using Regression and MachineLearning Technology",Elseveir,IERIProcedia 6 ( 2014 ) 52 - 56.

15. SaeedAzfar, Adnan Nadeem, Abdul Basit, 2015." Pest detection and control techniques usingwireless sensor network: A review", Journal of Entomology and Zoology Studies.

16. Jiangui Liu, John R. Miller, DrissHaboudane, Elizabeth Pattey,2004. "Exploring the Relationship between Red Edge Parameters and Crop Variables for Precision Agriculture", DOI: 10.1109/IGARSS.2004.1368649 · Source: IEEE Xplore, Research Gate.

17. A. K. Tripathy, J. Adinarayana, S. N. Merchant, U. B. Desai, K. Vijayalakshmi, D. RajiReddy, S. Ninomiya, M. Hirafuji, T. Kiura, 2012. "Data Mining and Wireless Sensor Network for Groundnut Pest Thrips Dynamics and Predictions ",Journal of Emerging Trends in Computing and Information Sciences, VOL. 3, NO. 6, ISSN 2079-8407.

18. Dr.NavneetGoyal, "Data Mining Applications in Agriculture", Department of Computer Science and Information Systems, BITS, Pilani.

19. D Ramesh, B Vishnu Vardhan, 2013, "Data Mining Techniques and Applications to Agricultural Yield Data", International Journal of Advanced Research in Computer and Communication Engineering, Vol. 2, Issue 9.

20. Sally Jo Cunningham, Geoffrey Holmes, "Developing Innovative applications in agriculture using data mining", Department of Computer Science, University of Waikato Hamilton, New Zealand.

21. JayGholap, AnuragIngole, JayeshGohil, ShaileshGargade, Vahida Attar, "Soil Data Analysis Using Classification Techniques and Soil Attribute Prediction", Dept. of Computer Engineering and IT, College of Engineering,Pune.

22. SitiKhairunniza-Bejo, SamihahMustaffha and Wan Ishak Wan Ismail,2014,"Application of Artificial Neural Network in Predicting Crop Yield: A Review", Journal of Food Science and Engineering 4 (2014) 1-9.

23. SidikMulyono, Mohamad Ivan Fanany, T. Basaruddin, 2012, "A Paddy Growth Stages Classification Using MODIS Remote Sensing Images with Balanced Branches Support Vector Machines", ICACSIS 2012 ISBN: 978-979-1421-15-7.

24. ManpreetKaur,HeenaGulati,Harish Kundra, 2014."Data Mining in Agriculture on Crop Price Prediction: Techniques and Applications", International Journal of Computer Applications (0975 - 8887) Volume 99- No.12. 
25. Hetal Patel, Dharmendra Patel,2014."A Brief survey of Data Mining Techniques Applied to Agricultural Data", International Journal of Computer Applications (0975 - 8887) Volume 95- No. 9.

26. Robert J. McQueen, Stephen R. Garner,Craig G. Nevill-Manning,Ian H. Witten "Applying Machine Learning to Agricultural Data", Management Systems, University of Waikato, Hamilton, New Zealand.

27. JharnaMajumdar, SnehaNaraseeyappa,ShilpaAnkalaki, "Analysis of agriculture data using data mining techniques: application of big data", Journal of Big data.

28. Elpiniki I. Papageorgiou, AthanasiosMarkinos, Theofanis Gemptos, 2009. "Application of fuzzy cognitive maps for cotton yield management in precision farming", Elsevier.

29. Latika Sharma, Nitu Mehta, 2012. "Data Mining Techniques: A Tool For Knowledge Management System In Agriculture",INTERNATIONAL JOURNAL OF SCIENTIFIC \& TECHNOLOGY RESEARCH VOLUME 1, ISSUE 5.

30. SalvatoreRampone, Alessio Valente,2016." Prediction of seasonal temperature using soft computing techniques": application in Benevento (Southern Italy) area, Springer, J Ambient Intell Human Comput (2017) 8:147-154

31. "Nations Encyclopaedia"- India-Agriculture- http://www.nationsencyclopedia.com/economies/Asia-and-the-Pacific/IndiaAGRICULTURE.html.

32. "Integrated Pest Management Principles", 2017.United States Environmental Protection Agency, https://en.wikipedia.org/wiki/Integrated_pest_management.

33. "K-Means Clustering", A tutorial on Clustering Algorithms, https://home.deib.polimi.it/matteucc/Clustering/tutorial_html/kmeans.html

34. "Artificial Neural Network (ANN) in Machine Learning ", 2017. https://data-flair.training/blogs/artificial-neural-network.

35. Rahul Saxena, 2017. "Naive Bayes Classifier in Machine Learning", Data Aspirant.com

36. "Support Vector Machine",2017,https://en.wikipedia.org/wiki/Support_vector_machine

37. Prashanth Gupta, 2017. "Decision Trees ", Towards Data Science, https://towardsdatascience.com/decision-trees-in-machinelearning.

38. Jason Brownless, 2016. "Linear Regression for Machine learning", https://machinelearningmastery.com/linear-regressionfor-machine-learning.

39. "Genetic Algorithms in Machine Learning", 2001.Jonathan Shapiro, Advanced Course on Artificial Intelligence, Machine Learning and its Applications, pp 146-168.

40. M.G. Hill, P. G. Connolly, P. Reutemann, D. Fletcher, 2014. "The use of Data Mining to assist Crop Protection decisions on Kiwifruit in New Zealand", Elsevier, vol 108,pp 250-257

41. Oladapo J. Olakulehin, Elijah Olusayo Omidiora, 2014."A genetic algorithm approach to maximize crop yields and sustain soil fertility", Net Journal of Agricultural Science, vol 2(3), pp 94-103

42. Veerle Desmet, Lieven Eeckhout, and Koen De Bosschere, "Using Decision Trees to Improve Program-Based and ProfileBased Static Branch Prediction",

43. Amie L. Melnychuk, 2012. "Multi-Temporal Crop Classification Using a Decision Tree in a Southern Ontario Agricultural Region", 
44. Kefaya Qaddoum, 2014. "Modified Nä̈ve Bayes Based Prediction Modeling for Crop Yield Prediction", International Journal of Computer and Information Engineering Vol: 8, No: 1.

45. L. Peacock, S. worner, J. Pitt, 2007. "The application of artificial neural networks in plant protection", Wiley Online Library. Volume 37, Issue 2, Pages 277-282. 